\title{
Sugary beverages consumption and latent autoimmune diabetes in adults: systematic review and meta-analysis
}

\section{ABSTRACT}

Introduction. Sugary beverages consumption (SBC) has amplified globally. SBC is associated with and leads to obesity and chronic diseases, nonetheless the role of SBC in development of autoimmune disorders such as latent autoimmune diabetes in adults (LADA) has not been addressed adequately among the different ethnic groups. We conducted this meta-analysis to compare the random effect of SBC intake on the risk of development of LADA.

Methods. We scrutinized the MEDLINE database up until January 2019 for articles addressing the association between sugary beverages, coffee consumption and LADA. We found 6 studies all of them addressed the LADA. We have included them in the meta-analysis and compared the random effect of SBC from the uppermost to the lowermost quantiles parallel to the risk of LADA.

Results. According to the research conducted, and data extracted, which involved 15027 contributors and 1862 patients with LADA, the participants in the uppermost quantile of SBC intake (used 1-2 servings per day in most cases) were at risk of developing LADA

Address for correspondence:

Ahmed M. El-Malky, PhD (C), MPH, CPHQ, DTQM, MB. Bch.

King Saud University Medical City

Riyadh, Saudi Arabia

Po Box 7805 Riyadh 11472 code 94

Phone: 4670011, fax: 4672439, ext 9-2737

Mob.: 00966564495895

e-mail: aelmalky@ksu.edu.sa

Clinical Diabetology 2020, 9, 2, 118-127

DOI: $10.5603 /$ DK.2020.0007

Received: 16.08 .2019

Accepted: 12.12 .2019 more than those in the lowermost quantile ( $\leq 1$ serving per month) (odds ratio [OR] 1.37 [95\% Cl 1.23-1.52]). Conclusion. According to the meta-analysis results excessive SBC intake may increase the risk of development of latent autoimmune diabetes in adults. However, no definite conclusions could be drawn due to heterogeneous data from low quality researches and the analysis was based on observational and casecontrol studies only. (Clin Diabetol 2020; 9; 2: 118-127)

Key words: sugary beverages consumption, latent autoimmune diabetes in adults, systematic review, meta-analysis

\section{Introduction and background}

In almost 50 years sugary beverages consumption (SBC) has increased at an alarming rate worldwide. For instance, in the United States, from 1970 to 2006 SBC per each individual reised from 64.4 to $141.7 \mathrm{kcal} /$ /day, forming double or twice the increase [1]. Similar results have been revealed in Mexico, where presently more than $12 \%$ of total calorie intake was represented by SBC [2]. The rapid and dramatic increase of SBC in several developing republics where SBC has increased concurrently in relation with increasing rates of growth and urbanization. In the 2007 annual report, the Coca-Cola company shows that, the amount of SBC sold in India and China increased by $14 \%$ and $18 \%$ respectively in one year, indicative of the considerable upsurges in trade at the national level [3]. For clarification sugary beverages include carbonated sodas, energy drinks, sport drinks, juice drinks, sweetened tea, iced tea, fruit drinks, and vitamin drinks. Recent research has been 
updated to include sweetened coffee and alcoholic beverages as sugary sweetened beverages (SSBs) [4]. Sugary beverages are currently the main source of supplementary additional sugars in a typical American diet. They contain multiple sweeteners such as corn fructose, sucrose, fruit juice extracts, all of which have the same basic analogous metabolic drawbacks [5].

On the other hand, a drink that is a $100 \%$ pure and natural fruit juice and not mixed with extra sugars is not considered a sugary sweetened beverage. progressively, teams of researchers and institutes are calling for maximum reductions in SBC $[5,6]$. Results from significant prospective researches in epidemiology have demonstrated a reliable positive correlation between SBC and obesity among children and adults [7]. Furthermore evolving proof also proposes that habitual SBC is correlated with a higher risk of developing diabetes and other metabolic disorders [8]. SBC has been proven to be the cause of obesity due to their more added sugar and imperfect recompense for total calorie intake [7].

Due to the high amount of fast absorbable sugars such as fructose corn syrup and sucrose, in combination with the large amounts consumed, SBC might increase the risk of diabetes through obesity and by raising the dietary glycemic index, and insulin resistance, which contributes to $\beta$-cell dysfunction [9]. An increase in metabolic impacts of SBC may also cause elevated blood pressure and the buildup of visceral fatty tissue and ectopic adiposities due to high liver de novo lipogenesis [10], which will in turn lead to the development of more triglycerides, LDL and decrease the level of HDL. The correlation between SBC and LADA is less clear [11, 12], but current research proposes that SBC may elevate the risk of diabetes in hereditarily predisposed subjects [11]. Conceivable mechanisms for SBC participation in autoimmune pathogenesis involve prompted beta cell apoptosis [13], perhaps due to prompted oxidative stress, high glucose levels [14, 15] or an overwhelmed beta cell, probably because it is more visible and exposed to the body's immunity [16]. LADA is a form of diabetes combining the features of both of type 1 and type 2 diabetes. Besides the involvement of autoimmune indicators such as anti-glutamic acid decarboxylase antibodies (GADA) with LADA. The most common biochemical marker in LADA is a mild or moderate insulin resistance [17]. Therefore, it is likely that SBC may impact the risk of LADA by the pathogenesis related to autoimmune disorders or insulin resistance, but then again this is still unclear. $9 \%$ of all cases diagnosed as adult-onset diabetes was recognized as LADA [18], which is considered a mixture of different elements from different types of diabetes. After review of the available literature, we conducted a systematic review and a meta-analysis to observe the relationship between SBC and the risk of development of LADA.

\section{Research methodology and design Search of literature}

Guidelines of the PRISMA 2009 Statement have been adopted - step by step as we conducted our meta-analysis [19]. Pertinent, applicable and multi-ethnic researches written in the English were recognized and acknowledged by an in-depth and meticulous probing of the following databases: MEDLINE electronic database; Cochrane Library; PsycINFO - American Psychological Association; Embase; CAB Abstracts; Web of Science by Clarivate Analytics (formerly known as ISI Web of Knowledge); CINAHL Database, BIOSIS (King Saud University Medical City Library of Medicine, Salah, MD) for studies from 1983 to January 2019, which included SBC and sugar-sweetened beverage consumption, such as: (soft drinks, soda, carbonated drinks, sweetened coffee, iced tea, alcoholic beverages, fruit drinks, squashes, sports drinks, soda-pop, cordials, energy drinks, punch, vitamin water drinks and sugary lemonade) and the risk of LADA. We searched for keywords including those mentioned above as well as those combined with "auto antibodies", "autoimmune disorders of $\beta$-cells of the pancreas", "latent diabetes", "latent autoimmune diabetes in adults", and "LADA." We used this method as extensively as possible as our primary means of exploration and in the next successive medical subheading (MESH) terms examination. Every relevant article that was found, we followed its references, searching for any hints of another thread. We searched for references and cross-references that would possibly guide us to other references. We searched not only for articles published in journals, but also those in the press, books, magazines, newspapers, websites, documentary films, dissertations, congressional publications, international organizational reports, and even editorials by deploying the (Citation Machine ${ }^{\circledR}$ ) as a means of accomplishing our purpose and achieving our objective.

Due to the high possibility for confusing and converse causation, we have excluded cross-sectional researches. We have also excluded short-term trials as they were unable to address the long-term relationship that we are exploring. However these short-term studies do provide significant intuition about the possible causal biological mechanisms and thus has helped further our understanding of the causality in some capacity. 


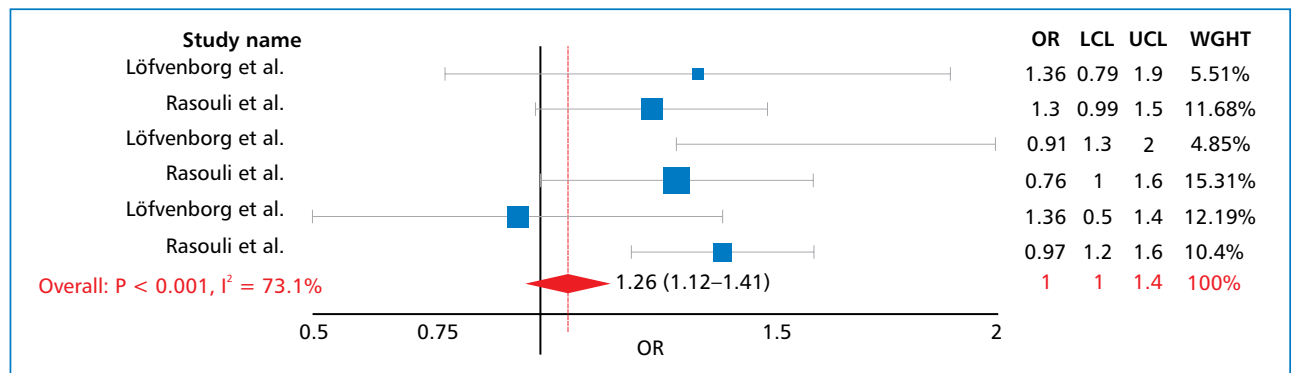

Figure 1A. Forest plot shows researches evaluating SBC and risk of LADA, comparing extreme quantiles of intake, random effects estimate

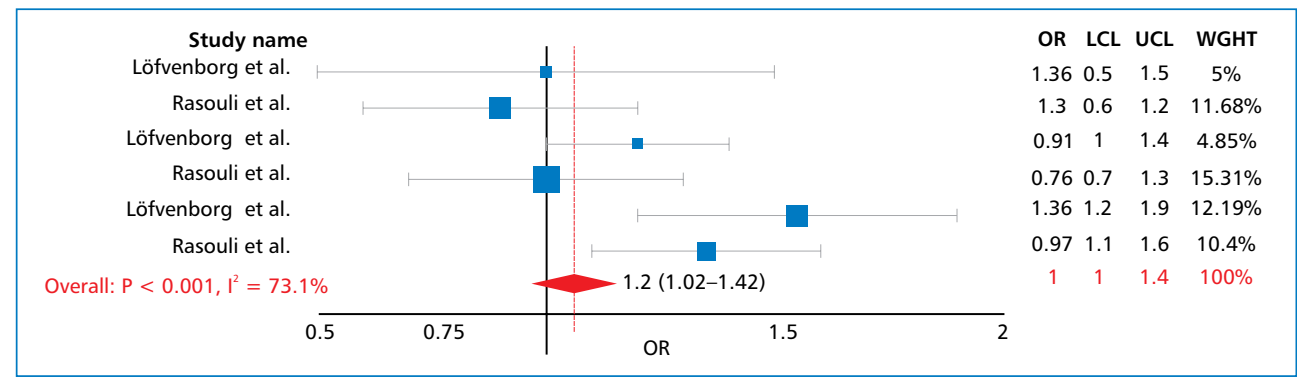

Figure 1B. Forest plot shows researches assessing SBC and type 2 diabetes mellitus comparing extreme quantiles of intake, random effects estimate

\section{Inclusion criteria and extracting data}

In our meta-analysis we included population based observational epidemiological studies as inclusion criteria. Criteria for inclusion comprised the end points of LADA, associated measure of variance (standard error or confidence interval) and relative risk as well as measures of SBC and potential mediators' adjustment. After we applied these criteria, our collected works selected eight identified articles out of 148 relevant references. Those 148 references were derived from 7534 citations (Figure 1). Each of the eight studies hit our target precisely [20-27]. Two of these studies have been excluded as one of them was review of literature [27] and the other was a master's thesis which was published as a paper later on, and selected from our target group [24].

The remaining six studies [20-27], all of which were held in Sweden, and written in English and those were two main weak points and mentioned as limitations. Only one study was done in the form of three cross sectional surveys on data from Nord-Trøndelag Health survey (HUNT) in 2012 [23]. The others were performed in the form of population based case-control studies, published in 2016-2019, apart from the two studies which were published in 2014 [21, 22]. All of the studies measure the association between SBC versus LADA, using age, sex, BMI, family history, total calorie intake, smoking, and education as potential mediators. Only two addressed the genetic susceptibility as an independent variable $[20,25]$. Estimated adjusted odds ratio (OR) of diabetes were entailed in relation to SBC. Standard errors and coefficients of variation were attained from Rasouli et al. [21] and Löfvenborg et al. [22] through subsequent communications. Two of the team members independently extracted the data. No variances were noticed in the extracted data to provide estimation of the effect, comparing skewed or drastic quantiles of SBC. All studies have defined one serving as $200 \mathrm{ml}$ however there are some notable variations in estimation of the serving size including Löfvenborg et al. [20] in which the maximum level of SBC was $200 \mathrm{ml}$ servings per day and lowest amount of intake was $200 \mathrm{ml}$ servings per week. With Rasouli et al. [21], the average intake of alcoholic beverages was 12 grams per day, while the maximum amount served was 25 grams per day. Löfvenborg et al. [22] established that the minimum amount of sweetened coffee per day was $<2$ cups while highest amount reached up to $>6$ cups. The minimum intake of alcohol per day with Rasouli et al. [23] was $<1$ time and $>10$ times in the highest category of intake. Löfvenborg et al. [24] classified the amount of servings of soft drinks and sodas as $(<1,1-2$ and $>2$ servings per day). Finally the least amount of coffee served with Rasouli et al. [25] was $<1$ cup per day and the highest was $>4$ cups. 


\section{Patient and public}

\section{involvement statement}

Our current study design was conducted in the form of meta-analysis and systematic research on already published study articles, so no patient involvement was documented, and the used materials was only published data.

\section{Limitations of the study}

The first limitation and point of weakness is that we conducted this meta-analysis and there was heterogeneity of results. All studies had different populations, different designs and outcomes. However all of them addressed the same topic and the same research question. Available literature regarding this topic is very scarce, in addition to heterogeneous data from low quality studies depending on retrospective, observational or case-control designs only. This in turn reflected negatively on the level of evidence and conclusion. Moreover, there were no prospective or long term experimental, interventional or randomized studies, with sufficient follow-up period, to demonstrate the potential relationship between sugary beverage consumption and LADA, and most probably such researches will never be conducted due to ethical reasons. The included studies were restricted to those published in English which lead to exclusion of non-English studies with their evidence base. This may increase the likelihood of selection bias. Also, there was point of limitation and unavoidable weaknesses. All of the studies were in Sweden. And we could do nothing to overcome all these point. Wide range of the dates of publications of the included studies, almost five decades, increases the validity and significance of the results.

\section{Analysis and investigation}

A total of six studies with nine data points are comprised in this meta-analysis of LADA and sugary beverages consumption [20-25]. We used STATA (version 9.0; Stata Corp, College Station, TX, USA) to attain instantaneous relative risks employing random effects models as well as fixed effects models designed from the logarithm of the relative risks and matching $95 \%$ confidence intervals of the separate studies [20-27]. A random-effects model was used primarily because it integrates the constituents of variance within the study itself and also between the studies. Egger's test was acknowledged to be employed in case of heterogeneity between studies and it's also considered to be the more conventional method [28]. We assessed the heterogeneity significance of the results throughout our selected studies by the application of Cochrane $\mathrm{Q}$ test, in spite of the presence of lack of sensitivity.
We followed Cochrane $Q$ test by an $1^{2}$ statistical analysis which embodies the proportion of whole disparity across studies because of inter-study heterogeneity [29]. Sensitivity analysis has been conducted to avoid heterogeneity, which might occur as a result of the total calorie intake modification which includes a follow up procedure and other potential mediators. We used these combined mediators as conjecturers and forecasters of effect in the meta-regression analyses. Those mediators were likely be able to influence the association between SBC and LADA, so we are therefore obliged to adjust all of these mediators to weaken and lessen the effect. We used a visual assessment of the Begg funnel plot and applied the Begg and Egger analysis to evaluate and appraise any possibility for publication bias [30, 31]. Generally case-control studies can study rare diseases which have multiple risk factors for one disease as they are relatively cheap, quick and easy to design due to retrospective recall because of the already existing data. However, this design could not study several diseases, rare exposures or even estimate the incubation period between risk factor of the disease in question, and disease itself. Neither could it measure the risk directly nor even the occurrence rates including the incidence and prevalence of the same. Relative risk could not be calculated but the Odds ratio could be. Thus given everything mentioned above, in terms of strength of association, case control studies showed the same strength as cohort because both were analytical studies [32].

\section{Results and findings}

Characteristics of all the study population included within our meta-analyses are presented in Table 1. Each research study assessed the risk of SBC in development of LADA (nine data points) [20-25], comprising males and females of the Caucasian population from Sweden and all of whom were adults. Regarding all the selected case-control studies, each of which were compared via a retrospective recall of previous exposure to the risk factor, which was sugary beverages consumption, including coffee and alcohol. Cases were matched with controls in relation to number of participants and demographic characteristics and was conducted by interviews and structured questionnaires which included food frequency questionnaires (FFQs). There were 15027 participants involved with and 1862 patients with LADA. The research articles assessed the dietary intake [20-25] revealed effect estimations that were not adjusted for total calorie intake or measures of BMI. According to the data from those six articles, the shared odds ratio [OR] for LADA was 1.37 [95\% Cl 1.23-1.52]). Overall $P$ value $0.001, I^{2}=73.1 \%$, for the 


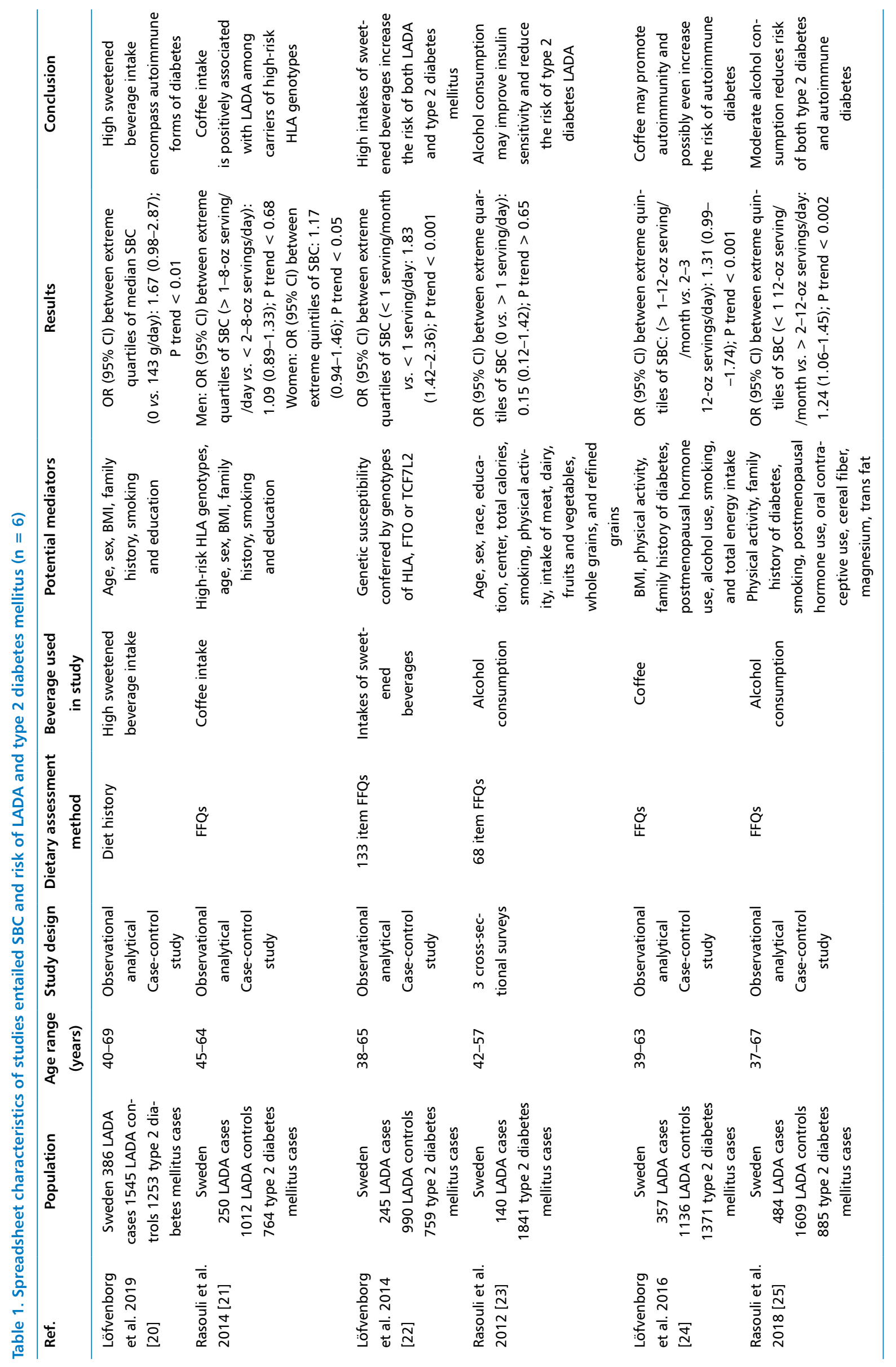


difference between extreme quantiles of SBC indicates an additional risk of $26 \%$ related with increased SBC.

Even though all researches apart from two [21, 23] exhibited positive associations and significant correlations between SBC and LADA, there was considerable heterogenetic difference among them in the analysis, where the $\mathrm{P}$ value was calculated for the test of heterogenetic difference for LADA: 12-66\% (95\% $\mathrm{Cl} 31-83)$, however $\mathrm{P}$ value, was 0.003 . Rasouli et al. [23] performed as a cross sectional study survey assessing LADA and expressed non-significant negative association [23]. If we exclude this research from our analysis, the heterogenetic difference will be reduced slightly, $12-62 \%$ (95\% Cl 17-82), however the P value, was 0.01 . On the other hand, the remaining studies showed clear significant positive association, except for one study [21] which also provided clear non-significant negative association between alcohol and LADA among men. However, it did conclude that alcohol could be a potential protective factor against LADA in women, due to the significant negative correlation $(P<0.68$ for men [21] $\mathrm{P}<0.05$ for women [21] $\mathrm{P}<0.65$ [23]). In spite of this condition, findings from the metaregression analysis did not find that the noted variation in either study $[21,23]$ to make a significant difference.

The case control study by Löfvenborg et al. [22], which displays a marginal significant positive correlation, has the smallest number of contributors and significantly lower amounts of SBC intake comparative to the other studies(median SBC is $143 \mathrm{~g} /$ day in uppermost quartile of intake, where intake of one 12-oz serving equal to $336 \mathrm{~g}$ ). Exclusion of this research from the analysis did not lessen heterogeneity, as was expected, given its low statistical significance and small proportion weight ( $P$ value, test for heterogenetic difference 0.002). However the research done by Rasouli et al. [25], which had the biggest significance and which used frequent measures of SBC, described the robust estimation. Exclusion of this research from the mutual analysis has decreased heterogenic difference to a marginal significance ( $\mathrm{P}$ value, test for heterogenic difference 0.05; $\mathrm{I}^{2}$ 51\% [95\% Cl 0-78\%]).

Assessments for publication bias usually depend on the supposition that a few studies with big variances may be more susceptible to publication bias, in comparison to large research studies. A visual review of the Begg funnel plot (accompanying Fig. 2), where by the standard error of log the relative risk (putting in consideration the study size) from each research was strategized against the log relative risk (effect of treatment), exhibited balance about the plot, suggestive of an impossible bias of publication, even though values for LADA may not be mostly help-

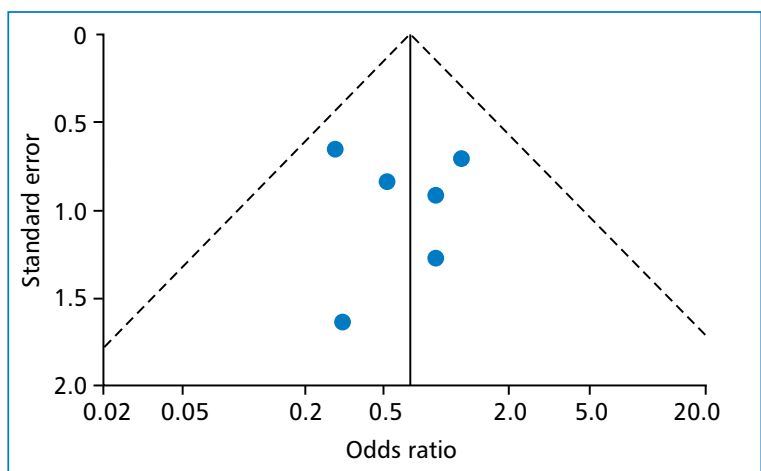

Figure 2. Funnel plot for assessment of publication bias

ful and informative because of the small number of studies comprised within the analysis. Studies with a big standard of error and great effect may recommend the existence of what is called "a small-study effect". In other words, the propensity of small research studies in the meta-analysis to show the big treatment effects) ( $P$ value for LADA was 0.75 in the studies of both Begg and Egger [28, 30, 31], Fig. 2).

Findings from our analysis of sensitivity in which both calorie, and BMI, adjusted coefficients were omitted $[20,22]$ revealed a slight escalation in risk of LADA with a pooled relative risk of 1.28 and $95 \%$ $\mathrm{Cl}$ (1.13-1.45). This is with regards to the randomeffects-model and on the other hand, relative risk of 1.250 (1.18-1.34) regarding the fixed-effects-model. There was a larger increase which was distinguishable in the dose-response-meta-analysis and when we excluded those studies [20, 22]: relative risk was 1.350 (1.14-1.59) and this regarding the random-effectsmodel. On the other hand, the relative risk of 1.180 (1.12-1.24) with regards to the fixed-effects-model. However findings from the meta-regression did not adjust for calorie intake as it was not considered to be an important mediator of outcome $(P=0.380)$. Further analysis of sensitivity was not conceivable for studies of LADA because they are too scarce and yet, both studies that did adjust for those mediators of outcome had borderline insignificant associations [20, 22], whilst the research that had shown unadjusted estimations also showed a significant positive correlation [21].

\section{Discussion and conclusion}

As can be deduced from the presented metaanalysis there is a clear association between SBC and risk of LADA. This is based upon the coefficients from five case-control studies and one cross sectional survey, which involved 15027 contributors and 1862 patients with LADA. Contributors in the uppermost group of SBC 


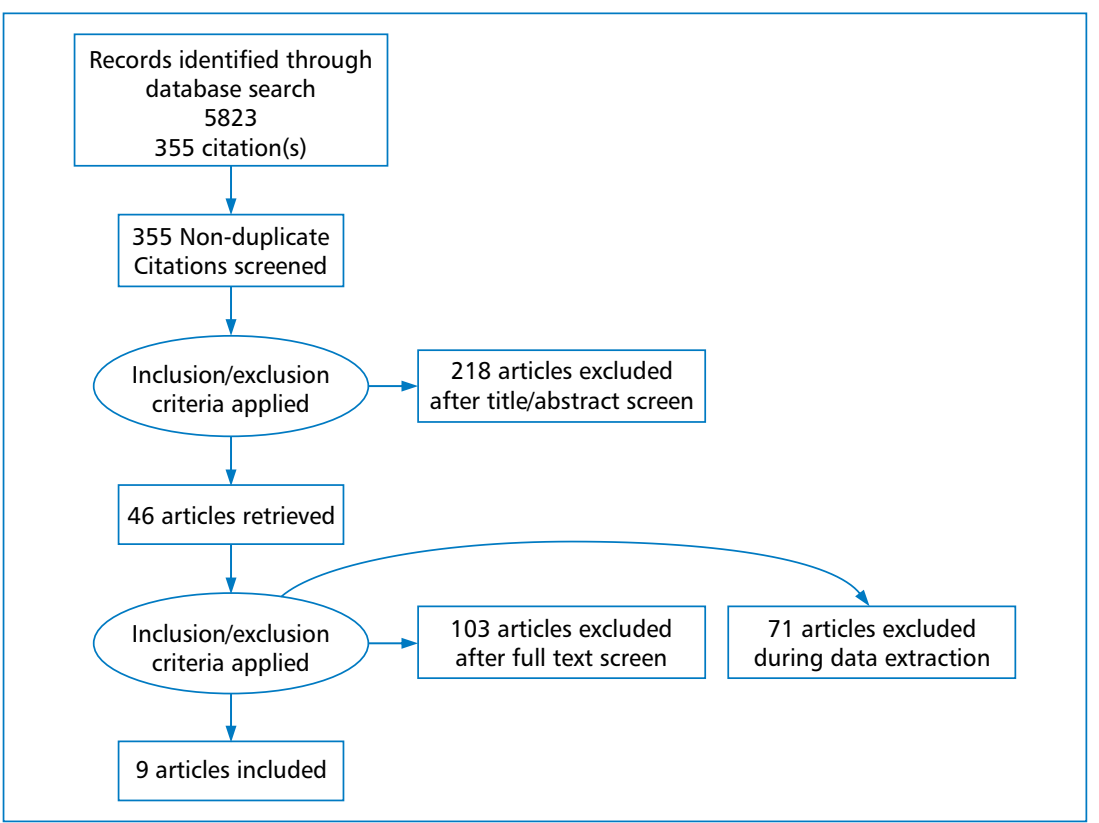

Figure 3. PRISMA diagram for selected studies

intake had a $20 \%$ more risk of developing LADA than those in the lowermost group of SBC intake.

Since we matched the extreme quantiles of SBC, mostly zero or one serving per month against one or two servings per day, groups of intake between the studies were not consistent or homogenous. Consequently, it is likely that a random biased classification fairly weakened the mutual estimation; though, findings were analogous to the dose-response-analysis, which used data from all groups. For those studies that did not outline a size of serving, an average serving of $(12 \mathrm{Oz}$ ) was presumed, which may overestimate or undervalue the experiential SBC levels but ought not to substantially disturb our results. Certainly there is considerable difference in study designs and assessment of exposure, through the studies, which may elucidate the observable notch between heterogenic differences in studies we perceived. Meta-analysis is integrally not as strong as the distinct prospective cohort research but it is still beneficial in providing a holistic view about the effect size. Moreover, they also provide larger investigations and studies with less random disparities and more weight than the smaller studies. Publication bias is always seen as a prospective apprehension especially with meta-analyses. Nevertheless standard assessments and visual scrutiny of the funnel plots garnered no proof of any publication bias in our analysis.

All the research that was involved in our metaanalysis included well-thought-out adjustments for possible confusion by several factors such as diet and lifestyle, and mostly due to the persistence of positive association, signifying an autonomous effect of SBC. High levels of SBC could be indicator of a generally unhealthy diet as they lean towards the inclusion of other factors such as, ingestion of high saturated and trans-fatty acids and a low fiber intake [12]. So, an imperfect adjustment for several diet and lifestyle factors could possibly overstate the strength of the positive correlation between SBC and risk of LADA. However, the consistency of results from these different studies decreases the probability that an enduring variable is responsible for the results. Longitudinal studies assessing diet and the risk of chronic disease may similarly be exposed to inverse causality, i.e., persons alter their diet due to subclinical disease symptoms or associated obesity, which may result in false associations [26]. Though it is not imaginable to totally remove these factors, studies with long periods of follow up and frequent measurements of nutritional intake have a tendency to be less susceptible to this process.

In a few studies, LADA was evaluated by selfassessment; yet, it has been demonstrated in confirmation studies that self-reporting of LADA is highly precise according to the review of medical records [26]. The bulk of research studies have used validated Food Frequency Questionnaire to assess SBC, which is the strongest technique for assessing a personal average dietary consumption associated with other valuation methods such as the 24 hour dietary recall [27]. However, errors of measurement in dietary assessment are always unavoidable, but because the studies we deliberated are case-control in design, faulty classifica- 
tion of SBC perhaps does not vary by case status. This non differential faulty classification of exposure may undervalue the real association between SBC and risk of such consequences.

SBC are thought to cause obesity due to their high supplementary sugar content, low compensatory water intake, reduced satiety and inadequate compensatory reduction in calorie intake during mealtimes which causes a positive energy balance and thus the body stores the extra food as fats $[7,8]$. Even though, SBC increases the risk of LADA, partially due to their participation in weight gain, an autonomous effect may also come from the increased amounts of fast absorbable carbohydrates as extra sugars, used as flavors in beverages. The results by Löfvenborg et al. [20] suggested that nearly half of the consequences of SBC on LADA were arbitrated through obesity. In a recent longitudinal research which followed 88,000 females for 24 years, who were consuming 2 servings per day and had a $34 \%$ more risk of coronary insufficiency in comparison to occasional consumers after adjustment for other potential mediators (relative risk 1.35, and $95 \% \mathrm{Cl}$ of 1.1-1.7, where P-value < 0.01) [33]. Further adjustement of potential mediators like BMI and total calorie intake, weakened the associations, however they were still statistically significant, indicating that the effect of SBC is not fully mediated by those factors. SBC has been proven to increase blood sugar and insulin levels quickly and intensely [34] and if frequently used in big quantities, will undoubtedly lead to a high dietetic glycemic load. High glycemic load (GL) nutrition will lead to glucose intolerance and insulin resistance mostly among obese people [9] and can raise the levels of inflammatory mediators such as the C-reactive protein, which are associated with the risk of autoimmune beta-cell dysfunction [35]. Results from other studies showed that a high nutritional GL can also magnify the risk of acquiring cholesterol gallstones which are associated with autoimmune insulin resistance [36].

Endogenic compounds in sugary beverages, such as progressive glycation-end-products, created during the procedure of adding caramel to soda beverages like cola might also influence the patho-physiological mechanisms associated with diabetes [37]. Modifications in taste preferences and quality of diet, induced by SBC, might also circuitously participate in increasing the risk of development of diabetes [5]. Interim experimental researches recommend that fructose, which is an essential component of fructose/sucrose corn syrup in fairly equivalent amounts, may lead to predominantly metabolic adverse effects when compared with glucose. This is because fructose is otherwise metabolized to lipids inside the liver, causing increased biochemical processes of creating fatty acids from acetyl CoA that are formed from a number of different mechanisms within the hepatic cell, leading to high levels of triglycerides, decreased high density lipoproteins, development of dyslipidemia, and also insulin resistance [38]. In brief, this meta-analysis has not shown that excessive SBC is associated with the risk of development of LADA. It offers moderate evidence to support the restricted intake of these drinks and the use of healthy substitutes instead like water to which will decrease the risk of chronic diseases. Nevertheless, there were no long term randomized studies, with sufficient follow-up period, to show potential relationship between sugary beverages and LADA, and probably such studies will never be conducted due to ethical reasons. So, no definite conclusions could be drawn due to heterogeneous data from low quality researches and the analysis was based on observational and case-control studies only. So the authors were urged to elaborate on the fact that no definite conclusions could be drawn.

\section{Summary}

Only six research papers worldwide addressed the relation between consumption of sugary beverages and the development of latent autoimmune diabetes in adults. These articles had contradictory findings regarding this risk factor. We conducted a systematic review and meta-analysis to establish statistical significance across studies that might otherwise seem to have conflicting results. This will increase the validity and reliability of information and any observed differences. This is the first systematic review and meta-analysis comparing this correlation, to find a clear significant association between sugary beverages consumption and latent autoimmune diabetes in adults.

\section{Ethical approval and consent to participate}

The authors certify that the guidelines of the PRISMA 2009 statement have been adopted.

\section{Competing interests and conflict-of-interest statement}

The authors certify that they have no conflicts of interest to declare including but not limited to financial, consultancy, advisory, institutional, and other relationships that might lead to a possible bias or misconstrue the results and/or conclusions of this research.

\section{Funding}

The authors certify that they have no funding source. This research received no specific grant from any funding agency in the public, commercial or notfor-profit sectors. 


\section{REFERENCES}

1. Popkin BM. Patterns of beverage use across the lifecycle. Physiol Behav. 2010; 100(1): 4-9, doi: 10.1016/j.physbeh.2009.12.022, indexed in Pubmed: 20045423.

2. Barquera S, Hernandez-Barrera L, Tolentino ML, et al. Energy intake from beverages is increasing among Mexican adolescents and adults. J Nutr. 2008; 138(12): 2454-2461, doi: 10.3945/ jn.108.092163, indexed in Pubmed: 19022972.

3. Last M. Caravans of Kola: The Hausa Kola Trade 1700-1900, by Paul LovejoyCaravans of Kola: The Hausa Kola Trade 1700-1900, by Paul Lovejoy. Zaria, Ahmadu Bello University Press, 1980. x, 181 pp. Canadian Journal of History. 1984; 19(3): 437-440, doi: 10.3138/cjh.19.3.437

4. Anjum I, Jaffery SS, Fayyaz M, et al. Sugar beverages and dietary sodas impact on brain health: a mini literature review. Cureus. 2018; 10(6): 127-130, doi: 10.7759/cureus.2756, indexed in Pubmed: 30094113.

5. Bodo YLe, Paquette MC, Wals PDe. Sugar-Sweetened beverage taxation as a public health policy instrument. Taxing Soda for Public Health. 2016: 59-74, doi: 10.1007/978-3-319-33648-0 3.

6. Johnson R, Appel L, Brands M, et al. Dietary Sugars Intake and Cardiovascular Health. Circulation. 2009; 120(11): 1011-1020, doi: 10.1161/circulationaha.109.192627

7. Malik VS, Schulze MB, Hu FB. Intake of sugar-sweetened beverages and weight gain: a systematic review. Am J Clin Nutr. 2006; 84(2): 274-288, doi: 10.1093/ajcn/84.1.274, indexed in Pubmed: 16895873.

8. Malik VS, Popkin BM, Bray GA, et al. Sugar-sweetened beverages, obesity, type 2 diabetes mellitus, and cardiovascular disease risk. Circulation. 2010; 121(11): 1356-1364, doi: 10.1161/CIRCULATIONAHA.109.876185, indexed in Pubmed: 20308626.

9. Schulze MB, Liu S, Rimm EB, et al. Glycemic index, glycemic load, and dietary fiber intake and incidence of type 2 diabetes in younger and middle-aged women. Am J Clin Nutr. 2004; 80(2): 348-356, doi: 10.1093/ajen/80.2.348, indexed in Pubmed: 15277155.

10. Stanhope KL, Schwarz JM, Keim NL, et al. Consuming fructosesweetened, not glucose-sweetened, beverages increases visceral adiposity and lipids and decreases insulin sensitivity in overweight/ /obese humans. J Clin Invest. 2009; 119(5): 1322-1334, doi: 10.1172/JCl37385, indexed in Pubmed: 19381015

11. Lamb MM, Frederiksen B, Seifert JA, et al. Sugar intake is associated with progression from islet autoimmunity to type 1 diabetes: the Diabetes Autoimmunity Study in the Young. Diabetologia. 2015; 58(9): 2027-2034, doi: 10.1007/s00125-015-3657-x, indexed in Pubmed: 26048237.

12. Pundziute-Lyckå A, Persson LA, Cedermark G, et al. Diet, growth, and the risk for type 1 diabetes in childhood: a matched casereferent study. Diabetes Care. 2004; 27(12): 2784-2789, doi: 10.2337/diacare.27.12.2784, indexed in Pubmed: 15562185.

13. Eizirik DL, Darville MI. beta-cell apoptosis and defense mechanisms: lessons from type 1 diabetes. Diabetes. 2001; 50 Suppl 1: S64-S69, doi: 10.2337/diabetes.50.2007.s64, indexed in Pubmed: 11272205.

14. Shao C, Gu J, Meng X, et al. Systematic investigation into the role of intermittent high glucose in pancreatic beta-cells. Int J Clin Exp Med. 2015; 8(4): 5462-5469, indexed in Pubmed: 26131124.

15. Kohnert KD, Freyse EJ, Salzsieder E. Glycaemic variability and pancreatic $\beta$-cell dysfunction. Curr Diabetes Rev. 2012; 8(5): 345-354, doi: 10.2174/157339912802083513, indexed in Pubmed: 22698079

16. Björk E, Kämpe $O$, Karlsson FA, et al. Glucose regulation of the autoantigen GAD65 in human pancreatic islets. J Clin Endocrinol Metab. 1992; 75(6): 1574-1576, doi: 10.1210/jcem.75.6.1464667, indexed in Pubmed: 1464667.

17. Tuomi T, Santoro N, Caprio S, et al. The many faces of diabetes: a disease with increasing heterogeneity. The Lancet. 2014; 383(9922): 1084-1094, doi: 10.1016/s0140-6736(13)62219-9.
18. Hawa $\mathrm{MI}$, Kolb $\mathrm{H}$, Schloot $\mathrm{N}$, et al. Action LADA consortium Adult-onset autoimmune diabetes in Europe is prevalent with a broad clinical phenotype: Action LADA 7. Diabetes Care. 2013; 36(4): 908-913, doi: 10.2337/dc12-0931, indexed in Pubmed: 23248199.

19. Moher D, Shamseer L, Clarke M, et al. PRISMA-P Group. Preferred reporting items for systematic review and meta-analysis protocols (PRISMA-P) 2015 statement. Syst Rev. 2015; 4: 345-351, doi: 10.1186/2046-4053-4-1, indexed in Pubmed: 25554246.

20. Löfvenborg JE, Ahlqvist E, Alfredsson L, et al. Genotypes of HLA, TCF7L2, and FTO as potential modifiers of the association between sweetened beverage consumption and risk of LADA and type 2 diabetes. Eur J Nutr. 2019; 3: 23-34, doi: 10.1007/s00394-01901893-x, indexed in Pubmed: 30656477.

21. Rasouli B, Andersson T, Carlsson PO, et al. Alcohol and the risk for latent autoimmune diabetes in adults: results based on Swedish ESTRID study. Eur J Endocrinol. 2014; 171(5): 535-543, doi: 10.1530/EJE-14-0403, indexed in Pubmed: 25117461.

22. Löfvenborg JE, Andersson T, Carlsson PO, et al. Coffee consumption and the risk of latent autoimmune diabetes in adults results from a Swedish case-control study. Diabet Med. 2014; 31(7): 799-805, doi: 10.1111/dme.12469, indexed in Pubmed: 24750356.

23. Rasouli B, Ahlbom A, Andersson T, et al. Alcohol consumption is associated with reduced risk of Type 2 diabetes and autoimmune diabetes in adults: results from the Nord-Trøndelag health study. Diabet Med. 2013; 30(1): 56-64, doi: 10.1111/j.14645491.2012.03713.x, indexed in Pubmed: 22612671.

24. Löfvenborg JE, Ahlqvist E, Alfredsson L, et al. Sweetened beverage intake and risk of latent autoimmune diabetes in adults (LADA) and type 2 diabetes. Eur J Endocrinol. 2016; 175(6): 605-614, doi: 10.1530/EJE-16-0376, indexed in Pubmed: 27926472.

25. Rasouli B, Ahlqvist E, Alfredsson L, et al. Coffee consumption, genetic susceptibility and risk of latent autoimmune diabetes in adults: A population-based case-control study. Diabetes Metab. 2018; 44(4): 354-360, doi: 10.1016/j.diabet.2018.05.002, indexed in Pubmed: 29861145.

26. Hu FB, Leitzmann MF, Stampfer MJ, et al. Physical activity and television watching in relation to risk for type 2 diabetes mellitus in men. Arch Intern Med. 2001; 161(12): 1542-1548, doi: 10.1001/archinte.161.12.1542, indexed in Pubmed: 11427103.

27. Sharif K, Watad A, Bragazzi NL, et al. Coffee and autoimmunity: More than a mere hot beverage! Autoimmun Rev. 2017; 16(7): 712-721, doi: 10.1016/j.autrev.2017.05.007, indexed in Pubmed: 28479483.

28. Egger M, Smith GD, Sterne J. 6.14 Systematic reviews and metaanalysis. Oxford Textbook of Public Health. 2009; 54: 671-682, doi: $10.1093 / \mathrm{med} / 9780199218707.003 .0039$.

29. Higgins JPT, Thompson SG. Quantifying heterogeneity in a metaanalysis. Stat Med. 2002; 21(11): 1539-1558, doi: 10.1002/ sim.1186, indexed in Pubmed: 12111919.

30. Begg CB, Mazumdar M. Operating characteristics of a rank correlation test for publication bias. Biometrics. 1994; 50(4): 1088-1101, indexed in Pubmed: 7786990.

31. Egger M, Davey Smith G, Schneider M, et al. Bias in meta-analysis detected by a simple, graphical test. BMJ. 1997; 315(7109): 629-634, doi: 10.1136/bmj.315.7109.629, indexed in Pubmed: 9310563.

32. Herkner $\mathrm{H}$, Male $\mathrm{C}$. Observational Studies. Clinical Pharmacology: Current Topics and Case Studies. 2016: 109-136, doi: 10.1007/978-3-319-27347-1_9.

33. Fung TT, Malik V, Rexrode KM, et al. Sweetened beverage consumption and risk of coronary heart disease in women. Am J Clin Nutr. 2009; 89(4): 1037-1042, doi: 10.3945/ajcn.2008.27140, indexed in Pubmed: 19211821

34. Janssens JP, Shapira N, Debeuf $P$, et al. Effects of soft drink and table beer consumption on insulin response in normal teenagers and carbohydrate drink in youngsters. Eur J Cancer Prev. 1999; 8(4): 289-295, doi: 10.1097/00008469-199908000-00004, indexed in Pubmed: 10493303. 
35. Liu S, Manson JE, Buring JE, et al. Relation between a diet with a high glycemic load and plasma concentrations of high-sensitivity C-reactive protein in middle-aged women. Am J Clin Nutr. 2002; 75(3): 492-498, doi: 10.1093/ajcn/75.3.492, indexed in Pubmed: 11864854.

36. Tsai CJ, Leitzmann MF, Willett WC, et al. Glycemic load, glycemic index, and carbohydrate intake in relation to risk of cholecystectomy in women. Gastroenterology. 2005; 129(1): 105-112, doi: 10.1053/j.gastro.2005.05.016, indexed in Pubmed: 16012940.
37. Uribarri J, Stirban A, Sander D, et al. Single oral challenge by advanced glycation end products acutely impairs endothelial function in diabetic and nondiabetic subjects. Diabetes Care. 2007; 30(10): 2579-2582, doi: 10.2337/dc07-0320, indexed in Pubmed: 17496238.

38. Bray GA. How bad is fructose? Am J Clin Nutr. 2007; 86(4): 895-896, doi: 10.1093/ajcn/86.4.895, indexed in Pubmed: 17921361. 